\title{
Fine Structure Analysis of the Configuration System of V II. Part I: Even-Parity Levels
}

\author{
Safa Bouazza1, Richard A. Holt², David S. Rosner2, Nathan M. R. Armstrong3 \\ ${ }^{1}$ LISM, Université de Champagne-Ardenne, Reims, France \\ ${ }^{2}$ Department of Physics and Astronomy, University of Western Ontario, London, Canada \\ ${ }^{3}$ Department of Physics and Astronomy, Mc Master University, Hamilton, Canada \\ Email: safa.bouazza@univ-reims.fr
}

Received 21 November 2013; revised 30 December 2013; accepted 15 January 2014

Copyright (C) 2014 by authors and Scientific Research Publishing Inc.

This work is licensed under the Creative Commons Attribution International License (CC BY). http://creativecommons.org/licenses/by/4.0/

(c) (i) Open Access

\section{Abstract}

Using a linked-parameter technique of level-fitting calculations in a multi configuration basis, a parametric analysis of fine structure (fs) for even-parity levels of V II, involving six configurations, has been performed. This led us to exchange the assignments of two triplets, $3 d^{3}\left({ }^{2} F\right) 4 s c^{3} F$ and $3 d^{4}$ $d^{3} \mathrm{~F}$, reported in earlier analyses as being located at $30,300 \mathrm{~cm}^{-1}$ and $30,600 \mathrm{~cm}^{-1}$, respectively. This is confirmed by experimental hyperfine structure (hfs) A constants, used as fingerprints. Moreover, the current singlet $3 d^{2} 4 s^{2}{ }^{1} D_{2}$ position is likely too high. The fs parameters, magnetic Landé g-factors, and the percentage of leading eigenvectors of levels are calculated. We present also predicted singlet, triplet and quintet positions for missing experimental levels up to 100,000 $\mathrm{cm}^{-1}$. The single-electron hfs parameters are determined in their entirety for ${ }^{51} \mathrm{~V}$ II for the model space $(3 d+4 s)^{4}$ with good accuracy. For the model space $(3 d+4 s)^{4}$ of ${ }^{51} \mathrm{~V}$ II the single-electron hfs parameters are computed; furthermore, our achieved theoretical evaluations of the single-electron hfs parameters, thanks to the use of $a b$ initio calculations, reinforce the validity of these hfs parameter values, deduced from experimental data.

\section{Keywords}

Fine Structure, Hyperfine Structure, Energy Levels, Ab-Initio Calculations, V II Spectrum

\section{Introduction}

The early successful fs analysis of the V II spectrum was due to Meggers and Moore [1]. According to their classification, all except two of the terms were known in the configurations $3 d^{3} 4 s$ and $3 d^{4}$, but $3 d^{2} 4 s^{2}$ was altogether unknown. Later, Sugar and Corliss compiled the energy levels of vanadium in its 23 stages of ionization, which were analysed from atomic spectra [2]. Iglesias [3] added 30 new levels to those already published for the 
$3 d^{3} 4 d$ configuration, using 149 lines, classified as $3 d^{3} 4 p-3 d^{3} 4 d$ transitions. Up to now, no parametric analysis of hfs exists for any even or odd configurations of V II. We propose to fill this absence, as we did previously for many singly ionized atoms: Hf II, Zr II, Ta II, Ti II and Nb II [4]-[8] in an aim to complete previous works and to eliminate erroneous level assignments. The background and motivation of this work should present high interest for astrophysical investigations, very useful in the study of the history of nucleosynthesis, chemically peculiar stars and the sun.

\section{Accurate Fine and Hyperfine Structure Analysis}

As only a few lowest energy configuration even-parity levels were available from experimental data, in our previous works regarding the fs of transition metal elements much of our analyses of model spaces were restricted to $(3 d+4 s)^{4},(4 d+5 s)^{4}$ and $(5 d+6 s)^{4}$. Fortunately, the energy levels of the five lowest configurations for V II are determined experimentally [1] [3]. We also know that there is poor isolation of the configurations $(3 d+4 s)^{4}$ from other configurations in the $3 d$-elements since some of their levels are located above the levels belonging to other even-parity configurations whose centers of gravity positions are higher.

Therefore, we use a configuration basis set, called the extended model space, which consists of the following six configurations: $(3 d+4 s)^{4}+3 d^{3} 5 s+3 d^{3} 4 d+3 d^{3} 5 d$. The interactions between particular states are determined quantitatively by this analysis. The complete details of fs analysis were already given in our previous papers: see for instance [8].

The fs least squares fitting procedure has been carried out for over 170 energy levels attributed to the extended model space. Table 1 lists the observed energy levels, calculated eigenvalues, and percentages of the largest and next largest wave function components with the corresponding LS term designations. A set of 37 parameters selected among a total of 157 , requisite for fs analysis, treated as free, concern only configurations with known experimental levels, i.e. $3 d^{2} 4 s^{2}, 3 d^{3} 4 s, 3 d^{4}, 3 d^{3} 4 d$ and $3 d^{3} 5 s$ in this work.

The fitted values of these parameters are given in Table 2 and Table 3 with their uncertainties in parentheses; the agreement was improved by taking into account the interactions between all known configuration energy levels. For comparison we have inserted also $a b$ initio calculations using the Cowan code [9]. A fit with a standard deviation of $55 \mathrm{~cm}^{-1}$ has been achieved. This fit may be considered as good, considering the large number of degrees of freedom: $132=169-37$. The other parameters with significant values are fixed to their weighted $a b$ initio values while those expected to be small, although predicted by theory, are fixed to zero and then are not listed in these two tables. We confirm on the whole the attributions to term designations given previously [1]-[3] except in two cases: two triplet positions, $3 d^{3}\left({ }^{2} F\right) 4 s \mathrm{c}{ }^{3} \mathrm{~F}$ and $3 d^{4} \mathrm{~d}^{3} \mathrm{~F}$, located at $30,300 \mathrm{~cm}^{-1}$ and $30600 \mathrm{~cm}^{-1}$. We propose to invert these two triplet positions as we did in Table 1. It has been brought immediately to our attention by experimental hfs data given in Table 1 of Ref. [10] since when comparing A values for $\mathrm{J}=2$ and $\mathrm{J}=$ 3 one can notice A values for $3 d^{3}\left({ }^{2} F\right) 4 s c^{3} \mathrm{~F}$ are smaller than those of $3 d^{4} \mathrm{~d}^{3} \mathrm{~F}$ and $3 d^{4} \mathrm{~b}{ }^{3} \mathrm{~F}$ which means broadly that the magnetic contribution of an s-contact-electron is less important than that of a d-electron. We propose moreover to correct the wrong position of the singlet $3 d^{2} 4 s^{2}{ }^{1} \mathrm{D}_{2}$ which must be rather lower than $3 d^{3} 4 s^{2} \mathrm{D} ;{ }^{3} \mathrm{D}$, i.e. $44,104(40) \mathrm{cm}^{-1}$ instead of $44,657 \mathrm{~cm}^{-1}$.

Let us point out that all parameters except spin-orbit $\zeta_{\text {nd }}$ and energies of configuration centers of gravity $\mathrm{E}_{\mathrm{av}}$ are weighted by a factor $0.7696=\frac{F^{2}(3 d, 3 d)(f s)}{F^{2}(3 d, 3 d)(a b-\text { initio })}=$, i.e. the ratio between Slater integrals $F^{2}(3 d, 3 d)$ for the $3 d^{3} 4 s$ configuration obtained thanks to the fs study and $a b$ initio calculations, as we did previously [4]-[8]. In Table 4 we give up to $100,000 \mathrm{~cm}^{-1}$ our predicted data for missing experimental energies for these five configurations, analysed in [1]-[3]. This will surely help further experimental V II work to complete this ion study.

Concerning the hfs analysis we follow the many-body parametrisation method [11] which allows us to take advantage of similarities between configuration interaction effects observed independently in spin-orbit and hyperfine splitting. The radial parameters $a_{n l}^{\kappa k}, b_{n l}^{\kappa k}, a_{i}$ and $b_{i}$ have been evaluated by fitting them to experimentally determined hfs constants A and B using the theoretical expressions (Equations (4) and (5) of [12] for instance).

In 2011, Armstrong, Rosner and Holt applied fast-ion-beam laser-fluorescence spectroscopy to measure with good accuracy the magnetic dipole hfs A constants of 24 even levels and 31 odd levels of ${ }^{51} \mathrm{~V}$ II [10] which are the first published data for hfs of this ion. 
Table 1. Comparison between the observed and calculated energy levels (in $\mathrm{cm}^{-1}$ ) and $\mathrm{g}_{\mathrm{J}}$-factors. For each state the parent terms are given immediately after the configuration label in columns $3 \& 4$.

\begin{tabular}{|c|c|c|c|c|c|}
\hline $\begin{array}{l}\text { Obs. energy level } \\
{[2]}\end{array}$ & $\begin{array}{l}\text { Calc. } \\
\text { eigenvalue }\end{array}$ & $\begin{array}{l}\text { Largest eigenvector } \\
\text { component (\%) }\end{array}$ & $\begin{array}{l}\text { Next largest eigenvector } \\
\text { component }\end{array}$ & Calc. $g_{J}$ & $\begin{array}{c}\text { Obs g } \\
{[1]}\end{array}$ \\
\hline \multicolumn{6}{|l|}{$\mathbf{J}=\mathbf{0}$} \\
\hline 0.00 & -37.25 & $99.60 \mathrm{C}^{5} \mathrm{D}$ & $0.29 \mathrm{M}^{4} \mathrm{~F},{ }^{5} \mathrm{D}$ & 0.00 & \\
\hline 11295.513 & 11366.99 & $53.92 \mathrm{C}^{3} \mathrm{P}$ & $37.54 \mathrm{C}^{3} \mathrm{P}$ & 0.00 & \\
\hline 19161.422 & 19128.46 & $95.13 \mathrm{~B}{ }^{2} \mathrm{P} ;{ }^{3} \mathrm{P}$ & $2.39 \mathrm{~B}{ }^{4} \mathrm{P} ;{ }^{3} \mathrm{P}$ & 0.00 & \\
\hline 19902.608 & 19803.77 & $63.38 \mathrm{C}^{1} \mathrm{~S}$ & $18.80 \mathrm{C}^{1} \mathrm{~S}$ & 0.00 & \\
\hline 20156.670 & 20101.10 & $75.22 \mathrm{~B}{ }^{4} \mathrm{P} ;{ }^{3} \mathrm{P}$ & $12.45 \mathrm{C}^{1} \mathrm{~S}$ & 0.00 & \\
\hline 32420.050 & 32464.18 & $52.32 \mathrm{C}^{3} \mathrm{P}$ & $41.44 \mathrm{C}^{3} \mathrm{P}$ & 0.00 & \\
\hline 48898.010 & 48892.84 & $96.44 \mathrm{~A}^{3} \mathrm{P}$ & $2.91 \mathrm{C}^{3} \mathrm{P}$ & 0.00 & \\
\hline 74949.580 & 74965.42 & $94.88 \mathrm{M}^{4} \mathrm{~F},{ }^{3} \mathrm{P}$ & $1.82 \mathrm{M}^{2} \mathrm{P} ;{ }^{3} \mathrm{P}$ & 0.00 & \\
\hline 76281.366 & 76278.52 & $91.57 \mathrm{M}^{4} \mathrm{~F},{ }^{5} \mathrm{D}$ & $5.34 \mathrm{M}{ }^{4} \mathrm{P} ;{ }^{5} \mathrm{D}$ & 0.00 & \\
\hline 81669.529 & 81693.78 & $98.90 \mathrm{~K}{ }^{4} \mathrm{P} ;{ }^{3} \mathrm{P}$ & $0.58 \mathrm{~K}^{2} \mathrm{P} ;{ }^{3} \mathrm{P}$ & 0.00 & \\
\hline 87230.300 & 87236.45 & $76.93 \mathrm{M}^{4} \mathrm{P} ;{ }^{3} \mathrm{P}$ & $13.06 \mathrm{M}^{2} \mathrm{P} ;{ }^{3} \mathrm{P}$ & 0.00 & \\
\hline \multicolumn{6}{|l|}{$\mathbf{J}=\mathbf{1}$} \\
\hline 36.102 & -0.56 & $99.62 C^{5} \mathrm{D}$ & $0.29 \mathrm{M}{ }^{4} \mathrm{~F},{ }^{5} \mathrm{D}$ & 1.501 & \\
\hline 2604.040 & 2585.86 & $99.95 \mathrm{~B}{ }^{4} \mathrm{~F} ;{ }^{5} \mathrm{~F}$ & $0.03 \mathrm{~B}^{2} \mathrm{D} ;{ }^{3} \mathrm{D}$ & 0.000 & \\
\hline 11514.784 & 11571.95 & $54.07 \mathrm{C}^{3} \mathrm{P}$ & $37.42 \mathrm{C}^{3} \mathrm{P}$ & 1.501 & 1.48 \\
\hline 13511.799 & 13545.15 & $99.75 \mathrm{~B}{ }^{4} \mathrm{P} ;{ }^{5} \mathrm{P}$ & $0.13 \mathrm{~B}{ }^{2} \mathrm{P} ;{ }^{3} \mathrm{P}$ & 2.502 & 2.39 \\
\hline 18269.514 & 18268.86 & $61.04 \mathrm{C}^{3} \mathrm{D}$ & $29.43 \mathrm{~B}{ }^{2} \mathrm{D} ;{ }^{3} \mathrm{D}$ & 0.509 & 0.49 \\
\hline 19166.314 & 19144.69 & $89.70 \mathrm{~B}{ }^{2} \mathrm{P} ;{ }^{3} \mathrm{P}$ & $6.51 \mathrm{~B}{ }^{4} \mathrm{P} ;{ }^{3} \mathrm{P}$ & 1.484 & 1.40 \\
\hline 20089.650 & 20095.10 & $81.72 \mathrm{~B}{ }^{4} \mathrm{P} ;{ }^{3} \mathrm{P}$ & $5.27 \mathrm{C}^{3} \mathrm{P}$ & 1.438 & 1.35 \\
\hline 20522.147 & 20534.46 & $44.22 \mathrm{~B}{ }^{2} \mathrm{D} ;{ }^{3} \mathrm{D}$ & $33.39 \mathrm{C}^{3} \mathrm{D}$ & 0.571 & 0.58 \\
\hline 22273.636 & 22272.534 & $96.87 \mathrm{~B}{ }^{2} \mathrm{P} ;{ }^{1} \mathrm{P}$ & $1.29 \mathrm{~B}{ }^{4} \mathrm{P} ;{ }^{3} \mathrm{P}$ & 0.999 & 0.97 \\
\hline 32299.257 & 32369.00 & $52.38 \mathrm{C}^{3} \mathrm{P}$ & $41.43 \mathrm{C}^{3} \mathrm{P}$ & 1.501 & 1.48 \\
\hline 44201.640 & 44200.00 & $76.60 \mathrm{~B}{ }^{2} \mathrm{D} ;{ }^{3} \mathrm{D}$ & $23.13 \mathrm{~B}{ }^{2} \mathrm{D} ;{ }^{3} \mathrm{D}$ & 0.499 & $0.50 ?$ \\
\hline 48975.700 & 48981.81 & $96.55 \mathrm{~A}^{3} \mathrm{P}$ & $2.85 \mathrm{C}^{3} \mathrm{P}$ & 1.501 & \\
\hline 69146.385 & 69130.36 & $99.91 \mathrm{~K}{ }^{4} \mathrm{~F} ;{ }^{5} \mathrm{~F}$ & $0.04 \mathrm{M}^{2} \mathrm{D} ;{ }^{3} \mathrm{D}$ & 0.000 & \\
\hline 72518.626 & 72485.99 & $99.20 \mathrm{M}^{4} \mathrm{~F} ;{ }^{5} \mathrm{P}$ & $0.38 \mathrm{~N}^{4} \mathrm{~F} ;{ }^{5} \mathrm{P}$ & 2.500 & \\
\hline 72839.345 & 72852.27 & $89.92 \mathrm{M}{ }^{4} \mathrm{~F} ;{ }^{5} \mathrm{~F}$ & $9.65 \mathrm{M}{ }^{4} \mathrm{~F} ;{ }^{3} \mathrm{D}$ & 0.059 & \\
\hline 73181.639 & 73195.11 & $87.78 \mathrm{M}{ }^{4} \mathrm{~F} ;{ }^{3} \mathrm{D}$ & $9.96 \mathrm{M}{ }^{4} \mathrm{~F} ;{ }^{5} \mathrm{~F}$ & 0.443 & \\
\hline 75080.541 & 75097.44 & $94.40 \mathrm{M}{ }^{4} \mathrm{~F} ;{ }^{3} \mathrm{P}$ & $1.82 \mathrm{M}^{2} \mathrm{P} ;{ }^{3} \mathrm{P}$ & 1.499 & \\
\hline 76322.694 & 76320.27 & $91.47 \mathrm{M}^{4} \mathrm{P} ;{ }^{5} \mathrm{D}$ & $5.38 \mathrm{M}{ }^{4} \mathrm{P} ;{ }^{5} \mathrm{D}$ & 1.501 & \\
\hline 80542.337 & 80549.73 & $99.36 \mathrm{~K}{ }^{4} \mathrm{~F} ;{ }^{5} \mathrm{P}$ & $0.31 \mathrm{~K}^{2} \mathrm{P} ;{ }^{3} \mathrm{P}$ & 2.500 & \\
\hline 81735.141 & 81751.39 & $98.55 \mathrm{~K}{ }^{4} \mathrm{P} ;{ }^{3} \mathrm{P}$ & $0.68 \mathrm{~K}{ }^{2} \mathrm{P} ;{ }^{1} \mathrm{P}$ & 1.498 & \\
\hline 84359.490 & 84352.76 & $39.36 \mathrm{M}{ }^{4} \mathrm{P} ;{ }^{3} \mathrm{D}$ & $37.06 \mathrm{M}^{4} \mathrm{P} ;{ }^{5} \mathrm{~F}$ & 0.318 & \\
\hline 85096.450 & 85089.15 & $77.90 \mathrm{M}^{4} \mathrm{P} ;{ }^{5} \mathrm{P}$ & $18.81 \mathrm{M}{ }^{4} \mathrm{P} ;{ }^{5} \mathrm{D}$ & 2.452 & \\
\hline 87184.950 & 87180.72 & $72.36 \mathrm{M}{ }^{4} \mathrm{P} ;{ }^{3} \mathrm{P}$ & $12.14 \mathrm{M}^{2} \mathrm{P} ;{ }^{3} \mathrm{P}$ & 1.468 & \\
\hline
\end{tabular}




\section{Continued}

\begin{tabular}{|c|c|c|c|c|c|}
\hline $\mathbf{J}=\mathbf{2}$ & & & & & \\
\hline 106.643 & 70.89 & $99.64 C^{5} \mathrm{D}$ & $0.29 \mathrm{M}{ }^{4} \mathrm{~F} ;{ }^{5} \mathrm{D}$ & 1.501 & \\
\hline 2687.208 & 2665.31 & $99.95 \mathrm{~B}{ }^{4} \mathrm{~F} ;{ }^{5} \mathrm{~F}$ & $0.02 \mathrm{~B}^{2} \mathrm{D} ;{ }^{3} \mathrm{D}$ & 1.000 & 0.97 \\
\hline 8640.362 & 8668.69 & $94.36 \mathrm{~B}{ }^{4} \mathrm{~F} ;{ }^{3} \mathrm{~F}$ & $4.45 \mathrm{C}^{3} \mathrm{~F}$ & 0.666 & 0.65 \\
\hline 11908.261 & 11977.54 & $54.12 \mathrm{C}^{3} \mathrm{P}$ & $37.09 \mathrm{C}^{3} \mathrm{P}$ & 1.501 & 1.49 \\
\hline 13490.883 & 13467.88 & $69.00 \mathrm{C}^{3} \mathrm{~F}$ & $23.80 \mathrm{C}^{3} \mathrm{~F}$ & 0.666 & 0.59 \\
\hline 13594.723 & 13623.51 & $99.58 \mathrm{~B}{ }^{4} \mathrm{P} ;{ }^{5} \mathrm{P}$ & $0.22 \mathrm{~B}{ }^{2} \mathrm{P} ;{ }^{3} \mathrm{P}$ & 1.834 & 1.78 \\
\hline 18293.871 & 18294.96 & $63.00 \mathrm{C}^{3} \mathrm{D}$ & $27.31 \mathrm{~B}{ }^{2} \mathrm{D} ;{ }^{3} \mathrm{D}$ & 1.172 & 1.13 \\
\hline 19132.791 & 19122.80 & $80.94 \mathrm{~B}{ }^{2} \mathrm{P} ;{ }^{3} \mathrm{P}$ & $13.70 \mathrm{~B}{ }^{4} \mathrm{P} ;{ }^{3} \mathrm{P}$ & 1.486 & 1.38 \\
\hline 20343.046 & 20335.14 & $68.38 \mathrm{~B}{ }^{4} \mathrm{P} ;{ }^{3} \mathrm{P}$ & $9.95 \mathrm{~B}^{2} \mathrm{D} ;{ }^{3} \mathrm{D}$ & 1.439 & 1.36 \\
\hline 20617.073 & 20627.27 & $36.11 \mathrm{~B}^{2} \mathrm{D} ;{ }^{3} \mathrm{D}$ & $20.10 \mathrm{C}^{3} \mathrm{D}$ & 1.213 & 1.25 \\
\hline 20980.927 & 20993.21 & $37.78 \mathrm{~B}{ }^{2} \mathrm{D} ;{ }^{1} \mathrm{D}$ & $32.50 \mathrm{C}{ }^{1} \mathrm{D}$ & 1.027 & 1.02 \\
\hline 25191.035 & 25150.22 & $36.51 \mathrm{C}^{1} \mathrm{D}$ & $33.78 \mathrm{~B}{ }^{2} \mathrm{D} ;{ }^{1} \mathrm{D}$ & 1.000 & 0.99 \\
\hline 30267.511 & 30257.99 & $38.55 \mathrm{C}^{3} \mathrm{~F}$ & $22.71 \mathrm{~B}^{2} \mathrm{~F} ;{ }^{3} \mathrm{~F}$ & 0.666 & 0.67 \\
\hline 30673.088 & 30669.40 & $76.09 \mathrm{~B}{ }^{2} \mathrm{~F} ;{ }^{3} \mathrm{~F}$ & $15.57 \mathrm{C}^{3} \mathrm{~F}$ & 0.666 & 0.67 \\
\hline 32040.635 & 32115.49 & $52.52 \mathrm{C}^{3} \mathrm{P}$ & $41.26 \mathrm{C}^{3} \mathrm{P}$ & 1.501 & 1.38 \\
\hline 37937.694 & 37933.75 & $76.22 \mathrm{~A}^{3} \mathrm{~F}$ & $18.91 \mathrm{C}^{3} \mathrm{~F}$ & 0.666 & \\
\hline $44657.941^{*}$ & 44105.45 & $60.81 \mathrm{~A}{ }^{1} \mathrm{D}$ & $22.76 \mathrm{C}{ }^{1} \mathrm{D}$ & 1.001 & \\
\hline 44159.460 & 44185.34 & $76.77 \mathrm{~B}^{2} \mathrm{D} ;{ }^{3} \mathrm{D}$ & $22.67 \mathrm{~B}^{2} \mathrm{D} ;{ }^{3} \mathrm{D}$ & 1.167 & 1.14 \\
\hline 47324.288 & 47302.16 & $70.03 \mathrm{~B}{ }^{2} \mathrm{D} ;{ }^{1} \mathrm{D}$ & $22.38 \mathrm{~B}{ }^{2} \mathrm{D} ;{ }^{1} \mathrm{D}$ & 1.001 & \\
\hline 49204.650 & 49190.71 & $95.74 \mathrm{~A}^{3} \mathrm{P}$ & $2.60 \mathrm{C}^{3} \mathrm{P}$ & 1.496 & \\
\hline 50951.660 & 50930.31 & $48.61 \mathrm{C}^{1} \mathrm{D}$ & $32.62 \mathrm{C}^{1} \mathrm{D}$ & 1.003 & \\
\hline 69228.318 & 69214.03 & $99.55 \mathrm{~K}{ }^{4} \mathrm{~F} ;{ }^{5} \mathrm{~F}$ & $0.38 \mathrm{~K}{ }^{4} \mathrm{~F} ;{ }^{3} \mathrm{~F}$ & 0.999 & \\
\hline 70415.542 & 70397.31 & $99.16 \mathrm{~K}{ }^{4} \mathrm{~F} ;{ }^{3} \mathrm{~F}$ & $0.38 \mathrm{~K}{ }^{4} \mathrm{~F} ;{ }^{5} \mathrm{~F}$ & 0.667 & \\
\hline 72674.924 & 72643.66 & $98.63 \mathrm{M}{ }^{4} \mathrm{~F} ;{ }^{5} \mathrm{P}$ & $0.49 \mathrm{M}{ }^{4} \mathrm{~F} ;{ }^{3} \mathrm{D}$ & 1.831 & \\
\hline 72878.056 & 72868.91 & $59.43 \mathrm{M}{ }^{4} \mathrm{~F} ;{ }^{5} \mathrm{G}$ & $36.08 \mathrm{M}{ }^{4} \mathrm{~F} ;{ }^{5} \mathrm{~F}$ & 0.604 & \\
\hline 73027.311 & 73026.02 & $55.47 \mathrm{M}^{4} \mathrm{~F} ;{ }^{5} \mathrm{~F}$ & $38.44 \mathrm{M}{ }^{4} \mathrm{~F} ;{ }^{5} \mathrm{G}$ & 0.738 & \\
\hline 73310.069 & 73294.71 & $90.74 \mathrm{M}^{4} \mathrm{~F} ;{ }^{3} \mathrm{D}$ & $4.04 \mathrm{M}{ }^{4} \mathrm{~F} ;{ }^{5} \mathrm{~F}$ & 1.162 & \\
\hline 75335.879 & 75355.16 & $94.09 \mathrm{M}{ }^{4} \mathrm{~F} ;{ }^{3} \mathrm{P}$ & $1.72 \mathrm{M}^{2} \mathrm{P} ;{ }^{3} \mathrm{P}$ & 1.500 & \\
\hline 75813.489 & 75805.22 & $92.33 \mathrm{M}{ }^{4} \mathrm{~F} ;{ }^{3} \mathrm{~F}$ & $2.96 \mathrm{M}^{2} \mathrm{G} ;{ }^{3} \mathrm{~F}$ & 0.668 & \\
\hline 76403.674 & 76403.25 & $91.33 \mathrm{M}^{4} \mathrm{~F} ;{ }^{5} \mathrm{D}$ & $4.73 \mathrm{M}{ }^{4} \mathrm{P} ;{ }^{5} \mathrm{D}$ & 1.501 & \\
\hline 80623.249 & 80633.56 & $98.95 \mathrm{~K}{ }^{4} \mathrm{P} ;{ }^{5} \mathrm{P}$ & $0.60 \mathrm{~K}^{2} \mathrm{P} ;{ }^{3} \mathrm{P}$ & 1.833 & \\
\hline 81914.328 & 81918.43 & $99.16 \mathrm{~K}{ }^{4} \mathrm{P} ;{ }^{3} \mathrm{P}$ & $0.21 \mathrm{~L}{ }^{4} \mathrm{P} ;{ }^{3} \mathrm{P}$ & 1.501 & \\
\hline 84406.210 & 84451.41 & $43.57 \mathrm{M}^{4} \mathrm{P} ;{ }^{3} \mathrm{D}$ & $26.93 \mathrm{M}^{2} \mathrm{G} ;{ }^{3} \mathrm{D}$ & 1.126 & \\
\hline 85045.572 & 85043.16 & 83.87 M ${ }^{4} \mathrm{P} ;{ }^{5} \mathrm{P}$ & $13.43 \mathrm{M}^{4} \mathrm{P} ;{ }^{5} \mathrm{D}$ & 1.812 & \\
\hline 86001.530 & 86003.05 & $29.26 \mathrm{M}^{4} \mathrm{P} ;{ }^{3} \mathrm{~F}$ & $26.59 \mathrm{M}^{2} \mathrm{G} ;{ }^{3} \mathrm{~F}$ & 0.787 & \\
\hline 87215.750 & 87224.76 & $74.09 \mathrm{M}^{4} \mathrm{P} ;{ }^{3} \mathrm{P}$ & $11.65 \mathrm{M}^{2} \mathrm{P} ;{ }^{3} \mathrm{P}$ & 1.490 & \\
\hline
\end{tabular}




\section{Continued}

\begin{tabular}{|c|c|c|c|c|c|}
\hline $\mathbf{J}=\mathbf{3}$ & & & & & \\
\hline 208.790 & 176.03 & $99.65 \mathrm{C}^{5} \mathrm{D}$ & $0.29 \mathrm{M}{ }^{4} \mathrm{~F} ;{ }^{5} \mathrm{D}$ & 1.501 & \\
\hline 2808.959 & 2784.06 & $99.95 \mathrm{~B}{ }^{4} \mathrm{~F} ;{ }^{5} \mathrm{~F}$ & $0.02 \mathrm{~B}{ }^{4} \mathrm{~F} ;{ }^{3} \mathrm{~F}$ & 1.251 & 1.20 \\
\hline 8842.050 & 8860.61 & $94.04 \mathrm{~B}{ }^{4} \mathrm{~F} ;{ }^{3} \mathrm{~F}$ & $4.72 \mathrm{C}^{3} \mathrm{~F}$ & 1.083 & 1.04 \\
\hline 13542.645 & 13515.81 & $67.97 \mathrm{C}^{3} \mathrm{~F}$ & $23.09 \mathrm{C}^{3} \mathrm{~F}$ & 1.079 & 1.06 \\
\hline 13741.640 & 13753.15 & $99.89 \mathrm{~B}{ }^{4} \mathrm{~F} ;{ }^{5} \mathrm{P}$ & $0.03 \mathrm{M}{ }^{4} \mathrm{~F} ;{ }^{5} \mathrm{P}$ & 1.668 & 1.62 \\
\hline 14461.748 & 14501.41 & $60.98 \mathrm{C}^{3} \mathrm{G}$ & $36.44 \mathrm{~B}{ }^{2} \mathrm{G} ;{ }^{3} \mathrm{G}$ & 0.754 & 0.74 \\
\hline 16340.981 & 16372.76 & $62.73 \mathrm{~B}^{2} \mathrm{G} ;{ }^{3} \mathrm{G}$ & $36.14 \mathrm{C}^{3} \mathrm{G}$ & 0.750 & 0.76 \\
\hline 18353.827 & 18330.97 & $68.46 \mathrm{C}^{3} \mathrm{D}$ & $24.53 \mathrm{~B}{ }^{2} \mathrm{D} ;{ }^{3} \mathrm{D}$ & 1.334 & 1.30 \\
\hline 20622.983 & 20668.95 & $52.95 \mathrm{~B}^{2} \mathrm{D} ;{ }^{3} \mathrm{D}$ & $30.24 C^{3} \mathrm{D}$ & 1.334 & 1.26 \\
\hline 26839.749 & 26638.09 & $90.37 \mathrm{C}^{1} \mathrm{~F}$ & $8.13 \mathrm{~B}{ }^{2} \mathrm{~F} ;{ }^{1} \mathrm{~F}$ & 1.000 & 0.97 \\
\hline 30306.389 & 30299.56 & $33.57 \mathrm{C}^{3} \mathrm{~F}$ & $32.42 \mathrm{~B}{ }^{2} \mathrm{~F} ;{ }^{3} \mathrm{~F}$ & 1.083 & 1.06 \\
\hline 30641.767 & 30647.98 & $65.93 \mathrm{~B}{ }^{2} \mathrm{~F} ;{ }^{3} \mathrm{~F}$ & $21.59 \mathrm{C}^{3} \mathrm{~F}$ & 1.083 & 1.05 \\
\hline 34228.852 & 34216.02 & $91.47 \mathrm{~B}{ }^{2} \mathrm{~F} ;{ }^{1} \mathrm{~F}$ & $8.11 \mathrm{C}^{1} \mathrm{~F}$ & 1.000 & 1.00 \\
\hline 38193.021 & 38185.64 & $77.69 \mathrm{~A}^{3} \mathrm{~F}$ & $17.89 \mathrm{C}^{3} \mathrm{~F}$ & 1.084 & \\
\hline 44098.473 & 44121.78 & 77.37 B ${ }^{2} \mathrm{D} ;{ }^{3} \mathrm{D}$ & $22.33 \mathrm{~B}{ }^{2} \mathrm{D} ;{ }^{3} \mathrm{D}$ & 1.334 & \\
\hline 69352.530 & 69341.00 & $99.36 \mathrm{~K}{ }^{4} \mathrm{~F} ;{ }^{5} \mathrm{~F}$ & $0.59 \mathrm{~K}{ }^{4} \mathrm{~F} ;{ }^{3} \mathrm{~F}$ & 1.250 & \\
\hline 70629.831 & 70616.64 & $98.96 \mathrm{~K}{ }^{4} \mathrm{~F} ;{ }^{3} \mathrm{~F}$ & $0.59 \mathrm{~K}{ }^{4} \mathrm{~F} ;{ }^{5} \mathrm{~F}$ & 1.084 & 1.06 \\
\hline 72448.600 & 72439.34 & $98.32 \mathrm{M}^{4} \mathrm{~F} ;{ }^{5} \mathrm{H}$ & $0.75 \mathrm{M}^{4} \mathrm{~F} ;{ }^{5} \mathrm{G}$ & 0.503 & \\
\hline 72908.997 & 72881.92 & $97.83 \mathrm{M}^{4} \mathrm{~F} ;{ }^{5} \mathrm{P}$ & $1.39 \mathrm{M}{ }^{4} \mathrm{~F} ;{ }^{3} \mathrm{D}$ & 1.663 & \\
\hline 72951.558 & 72937.71 & $77.16 \mathrm{M}^{4} \mathrm{~F} ;{ }^{5} \mathrm{G}$ & $44.68 \mathrm{M}^{4} \mathrm{~F} ;{ }^{5} \mathrm{~F}$ & 1.118 & \\
\hline 73146.343 & 73128.86 & $76.27 \mathrm{M}{ }^{4} \mathrm{~F} ;{ }^{5} \mathrm{~F}$ & $45.04 \mathrm{M}{ }^{4} \mathrm{~F} ;{ }^{5} \mathrm{G}$ & 1.046 & \\
\hline 73530.712 & 73517.55 & $94.14 \mathrm{M}^{4} \mathrm{~F} ;{ }^{3} \mathrm{D}$ & $1.82 \mathrm{M}{ }^{4} \mathrm{P} ;{ }^{3} \mathrm{D}$ & 1.337 & \\
\hline 75422.910 & 75412.58 & $92.32 \mathrm{M}^{4} \mathrm{~F} ;{ }^{3} \mathrm{G}$ & $2.20 \mathrm{M}^{2} \mathrm{G} ;{ }^{3} \mathrm{G}$ & 0.757 & \\
\hline 75966.119 & 75963.84 & $90.07 \mathrm{M}^{4} \mathrm{~F} ;{ }^{3} \mathrm{~F}$ & $3.05 \mathrm{M}^{2} \mathrm{G} ;{ }^{3} \mathrm{~F}$ & 1.077 & \\
\hline 76521.357 & 76522.23 & $91.27 \mathrm{M}{ }^{4} \mathrm{~F} ;{ }^{5} \mathrm{D}$ & $4.84 \mathrm{M}{ }^{4} \mathrm{P} ;{ }^{5} \mathrm{D}$ & 1.501 & \\
\hline 80782.426 & 80783.98 & $99.62 \mathrm{~K}{ }^{4} \mathrm{~F} ;{ }^{5} \mathrm{P}$ & $0.23 \mathrm{M}{ }^{4} \mathrm{P} ;{ }^{5} \mathrm{P}$ & 1.668 & \\
\hline 81263.626 & 81321.39 & $99.91 \mathrm{~K}^{2} \mathrm{G} ;{ }^{3} \mathrm{G}$ & $0.02 \mathrm{~L}^{2} \mathrm{G} ;{ }^{3} \mathrm{G}$ & 0.750 & \\
\hline 84459.916 & 84504.10 & $53.04 \mathrm{M}^{4} \mathrm{P} ;{ }^{3} \mathrm{D}$ & $27.87 \mathrm{M}^{2} \mathrm{G} ;{ }^{3} \mathrm{D}$ & 1.307 & \\
\hline 84643.381 & 84670.91 & 86.67 $\mathrm{M}^{2} \mathrm{G} ;{ }^{1} \mathrm{~F}$ & $7.33 \mathrm{M}^{2} \mathrm{G} ;{ }^{3} \mathrm{G}$ & 0.983 & \\
\hline 84999.355 & 85012.56 & $89.91 \mathrm{M}{ }^{4} \mathrm{P} ;{ }^{5} \mathrm{P}$ & $6.53 \mathrm{M}^{4} \mathrm{P} ;{ }^{5} \mathrm{D}$ & 1.655 & \\
\hline 85076.720 & 85088.67 & $82.85 \mathrm{M}^{2} \mathrm{G} ;{ }^{3} \mathrm{G}$ & $8.16 \mathrm{M}^{2} \mathrm{G} ;{ }^{1} \mathrm{~F}$ & 0.772 & \\
\hline 86113.793 & 86098.83 & $46.73 \mathrm{M}^{2} \mathrm{G} ;{ }^{3} \mathrm{~F}$ & $35.01 \mathrm{M}^{4} \mathrm{P} ;{ }^{3} \mathrm{~F}$ & 1.096 & \\
\hline 90381.370 & 90328.91 & $54.69 \mathrm{M}^{2} \mathrm{H} ;{ }^{1} \mathrm{~F}$ & $26.04 \mathrm{M}^{2} \mathrm{D} ;{ }^{1} \mathrm{~F}$ & 0.998 & \\
\hline 93806.100 & 93703.86 & $37.36 \mathrm{M}^{2} \mathrm{H} ;{ }^{1} \mathrm{~F}$ & $24.29 \mathrm{M}^{2} \mathrm{D} ;{ }^{1} \mathrm{~F}$ & 1.001 & \\
\hline
\end{tabular}




\section{Continued}

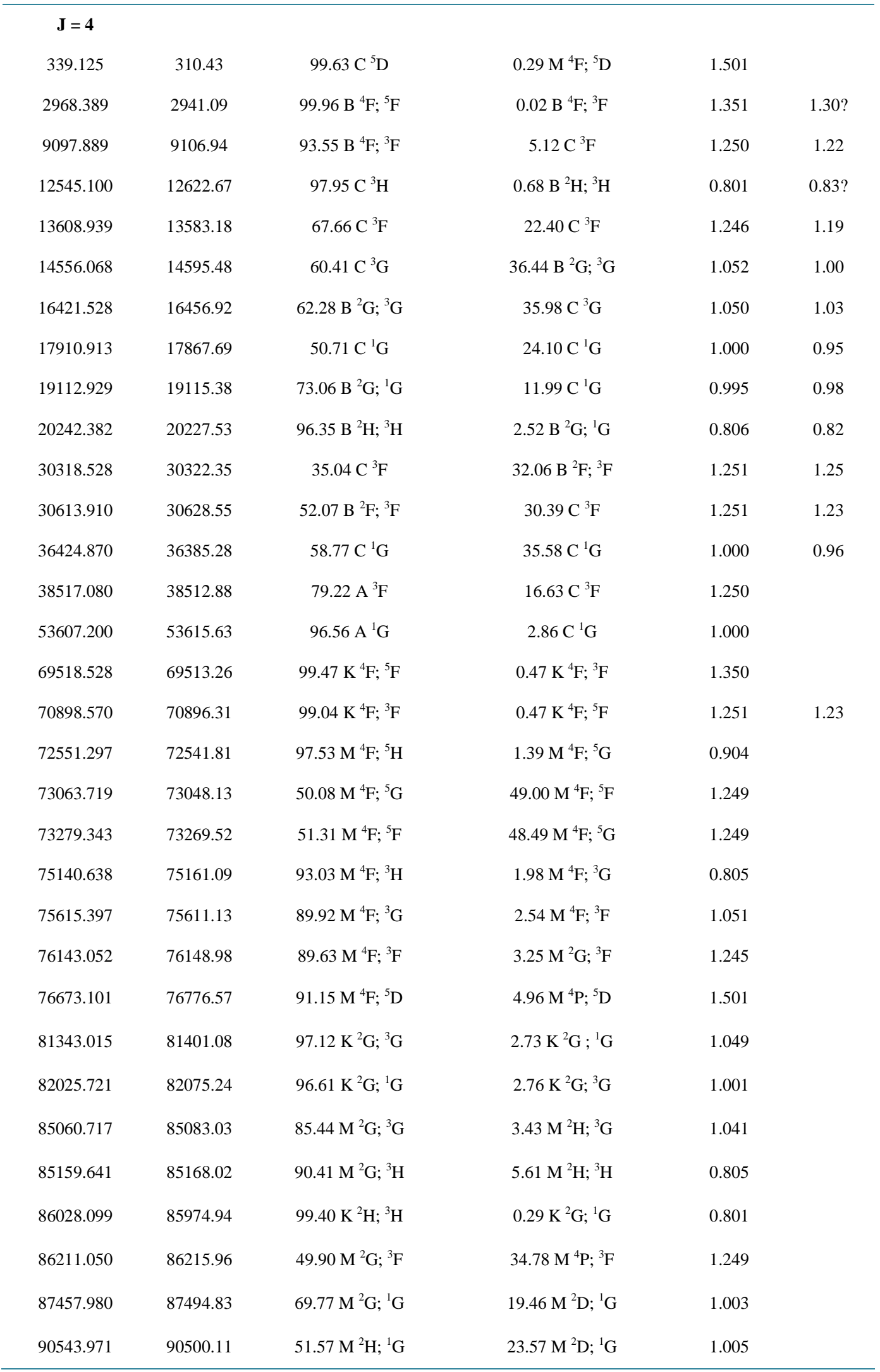




\section{Continued}

\begin{tabular}{|c|c|c|c|c|c|}
\hline $\mathbf{J}=\mathbf{5}$ & & & & & \\
\hline 3162.966 & 3129.47 & $99.95 \mathrm{~B}{ }^{4} \mathrm{~F} ;{ }^{5} \mathrm{~F}$ & $0.04 \mathrm{~B}{ }^{2} \mathrm{G} ;{ }^{3} \mathrm{G}$ & 1.401 & $1.28 ?$ \\
\hline 12621.485 & 12702.6 & $98.13 \mathrm{C}^{3} \mathrm{H}$ & $0.68 \mathrm{~B}{ }^{2} \mathrm{H} ;{ }^{3} \mathrm{H}$ & 1.034 & 1.02 \\
\hline 14655.607 & 14690.69 & $62.29 \mathrm{C}^{3} \mathrm{G}$ & 36.07 B ${ }^{2} \mathrm{G} ;{ }^{3} \mathrm{G}$ & 1.200 & 1.17 \\
\hline 16532.983 & 16569.48 & $62.99 \mathrm{~B}{ }^{2} \mathrm{G} ;{ }^{3} \mathrm{G}$ & $35.75 C^{3} \mathrm{G}$ & 1.200 & 1.16 \\
\hline 20280.251 & 20274.02 & $98.99 \mathrm{~B}{ }^{2} \mathrm{H} ;{ }^{3} \mathrm{H}$ & $0.68 \mathrm{C}^{3} \mathrm{H}$ & 1.034 & 1.01 \\
\hline 23391.150 & 23410.26 & $99.71 \mathrm{~B}{ }^{2} \mathrm{H} ;{ }^{1} \mathrm{H}$ & $0.12 \mathrm{~K}{ }^{2} \mathrm{H} ;{ }^{1} \mathrm{H}$ & 1.000 & 1.04 \\
\hline 69724.236 & 69729.28 & $99.91 \mathrm{~K}{ }^{4} \mathrm{~F} ;{ }^{5} \mathrm{~F}$ & $0.05 \mathrm{~K}{ }^{2} \mathrm{G} ;{ }^{3} \mathrm{G}$ & 1.401 & 1.39 \\
\hline 72680.856 & 72672.75 & $97.24 \mathrm{M}{ }^{4} \mathrm{~F} ;{ }^{5} \mathrm{H}$ & $1.65 \mathrm{M}{ }^{4} \mathrm{~F} ;{ }^{5} \mathrm{G}$ & 1.104 & \\
\hline 73223.351 & 73206.48 & $53.20 \mathrm{M}^{4} \mathrm{~F} ;{ }^{5} \mathrm{G}$ & $44.80 \mathrm{M}^{4} \mathrm{~F} ;{ }^{5} \mathrm{~F}$ & 1.326 & \\
\hline 73417.330 & 73417.60 & $53.98 \mathrm{M}{ }^{4} \mathrm{~F} ;{ }^{5} \mathrm{~F}$ & $44.70 \mathrm{M}{ }^{4} \mathrm{~F} ;{ }^{5} \mathrm{G}$ & 1.339 & \\
\hline 75346.306 & 75383.89 & $93.06 \mathrm{M}^{4} \mathrm{~F} ;{ }^{3} \mathrm{H}$ & $1.92 \mathrm{M}^{4} \mathrm{~F} ;{ }^{3} \mathrm{G}$ & 1.037 & \\
\hline 75854.219 & 75861.13 & $92.60 \mathrm{M}^{4} \mathrm{~F} ;{ }^{3} \mathrm{G}$ & $2.03 \mathrm{M}^{2} \mathrm{G} ;{ }^{3} \mathrm{G}$ & 1.197 & \\
\hline 81483.278 & 81541.61 & $99.50 \mathrm{~K}{ }^{2} \mathrm{G} ;{ }^{3} \mathrm{G}$ & $0.22 \mathrm{~K}{ }^{2} \mathrm{H} ;{ }^{3} \mathrm{H}$ & 1.200 & \\
\hline 84742.170 & 84771.02 & $97.89 \mathrm{M}^{2} \mathrm{G} ;{ }^{3} \mathrm{I}$ & $1.43 \mathrm{M}^{2} \mathrm{H} ;{ }^{3} \mathrm{I}$ & 0.833 & \\
\hline 84896.899 & 84927.93 & $90.38 \mathrm{M}^{2} \mathrm{G} ;{ }^{1} \mathrm{H}$ & $6.46 \mathrm{M}^{2} \mathrm{G} ;{ }^{3} \mathrm{G}$ & 1.004 & \\
\hline 85140.362 & 85162.11 & $84.54 \mathrm{M}^{2} \mathrm{G} ;{ }^{3} \mathrm{G}$ & $3.85 \mathrm{M}^{2} \mathrm{H} ;{ }^{3} \mathrm{G}$ & 1.192 & \\
\hline 85301.938 & 85308.03 & $82.79 \mathrm{M}^{2} \mathrm{G} ;{ }^{3} \mathrm{H}$ & $7.49 \mathrm{M}^{2} \mathrm{G} ;{ }^{1} \mathrm{H}$ & 1.035 & \\
\hline 86091.728 & 86039.72 & $97.94 \mathrm{~K}{ }^{2} \mathrm{H} ;{ }^{3} \mathrm{H}$ & $1.74 \mathrm{~K}{ }^{2} \mathrm{H} ;{ }^{1} \mathrm{H}$ & 1.033 & \\
\hline 86766.880 & 86695.84 & $96.71 \mathrm{~K}^{2} \mathrm{H} ;{ }^{1} \mathrm{H}$ & $1.82 \mathrm{~K}^{2} \mathrm{H} ;{ }^{3} \mathrm{H}$ & 1.001 & \\
\hline 88939.995 & 88915.30 & $97.24 \mathrm{M}^{2} \mathrm{H} ;{ }^{3} \mathrm{I}$ & $1.52 \mathrm{M}^{2} \mathrm{G} ;{ }^{3} \mathrm{I}$ & 0.834 & \\
\hline 89053.341 & 89027.95 & $92.39 \mathrm{M}^{2} \mathrm{H} ;{ }^{1} \mathrm{H}$ & $2.50 \mathrm{M}^{2} \mathrm{D} ;{ }^{3} \mathrm{G}$ & 1.008 & \\
\hline \multicolumn{6}{|l|}{$J=6$} \\
\hline 12706.078 & 12789.28 & $99.04 \mathrm{C}^{3} \mathrm{H}$ & $0.69 \mathrm{~B}^{2} \mathrm{H} ;{ }^{3} \mathrm{H}$ & 1.167 & $1.27 ?$ \\
\hline 19191.326 & 19194.69 & $99.15 \mathrm{C}^{1} \mathrm{I}$ & $0.45 \mathrm{M}^{2} \mathrm{H} ;{ }^{1} \mathrm{I}$ & 1.000 & $0.96 ?$ \\
\hline 20363.335 & 20362.12 & $99.21 \mathrm{~B}{ }^{2} \mathrm{H} ;{ }^{3} \mathrm{H}$ & $0.69 \mathrm{C}^{3} \mathrm{H}$ & 1.167 & 1.14 \\
\hline 72837.581 & 72833.32 & $97.72 \mathrm{M}^{4} \mathrm{~F} ;{ }^{5} \mathrm{H}$ & $1.25 \mathrm{M}^{4} \mathrm{~F} ;{ }^{5} \mathrm{G}$ & 1.216 & \\
\hline 73499.773 & 73440.60 & $98.00 \mathrm{M}{ }^{4} \mathrm{~F} ;{ }^{5} \mathrm{G}$ & $1.26 \mathrm{M}^{4} \mathrm{~F} ;{ }^{5} \mathrm{H}$ & 1.332 & \\
\hline 75592.481 & 75643.20 & $94.82 \mathrm{M}^{4} \mathrm{~F} ;{ }^{3} \mathrm{H}$ & $1.89 \mathrm{M}^{2} \mathrm{H} ;{ }^{3} \mathrm{H}$ & 1.167 & \\
\hline 84859.580 & 84883.73 & $97.83 \mathrm{M}^{2} \mathrm{G} ;{ }^{3} \mathrm{I}$ & $1.23 \mathrm{M}^{2} \mathrm{H} ;{ }^{3} \mathrm{I}$ & 1.024 & \\
\hline 85415.904 & 85440.02 & $91.53 \mathrm{M}^{2} \mathrm{G} ;{ }^{3} \mathrm{H}$ & $5.15 \mathrm{M}^{2} \mathrm{H} ;{ }^{3} \mathrm{H}$ & 1.166 & \\
\hline 86191.750 & 86143.63 & $99.86 \mathrm{~K}^{2} \mathrm{H} ;{ }^{3} \mathrm{H}$ & $0.05 \mathrm{M}^{2} \mathrm{G} ;{ }^{3} \mathrm{H}$ & 1.167 & \\
\hline 86453.764 & 86471.68 & $87.34 \mathrm{M}^{2} \mathrm{G} ;{ }^{1} \mathrm{I}$ & $10.63 \mathrm{M}^{2} \mathrm{H} ;{ }^{1} \mathrm{I}$ & 1.000 & \\
\hline 89005.580 & 89013.81 & $97.89 \mathrm{M}^{2} \mathrm{H} ;{ }^{3} \mathrm{I}$ & $1.26 \mathrm{M}^{2} \mathrm{G} ;{ }^{3} \mathrm{I}$ & 1.024 & \\
\hline 94371.80 & 94306.91 & $80.48 \mathrm{M}^{2} \mathrm{H} ;{ }^{1} \mathrm{I}$ & $8.89 \mathrm{M}^{2} \mathrm{G} ;{ }^{1} \mathrm{I}$ & 1.000 & \\
\hline \multicolumn{6}{|l|}{$\mathbf{J}=7$} \\
\hline 73021.143 & 73024.65 & $99.28 \mathrm{M}^{4} \mathrm{~F} ;{ }^{5} \mathrm{H}$ & $0.61 \mathrm{~N}{ }^{4} \mathrm{~F} ;{ }^{5} \mathrm{H}$ & 1.286 & \\
\hline 85004.880 & 85002.77 & $98.25 \mathrm{M}^{2} \mathrm{G} ;{ }^{3} \mathrm{I}$ & $1.01 \mathrm{M}^{2} \mathrm{H} ;{ }^{3} \mathrm{I}$ & 1.143 & \\
\hline 89082.769 & 89094.38 & $98.38 \mathrm{M}^{2} \mathrm{H} ;{ }^{3} \mathrm{I}$ & $0.99 \mathrm{M}^{2} \mathrm{G} ;{ }^{3} \mathrm{I}$ & 1.143 & \\
\hline \multicolumn{6}{|l|}{$\mathbf{J}=\mathbf{8}$} \\
\hline & 90180.86 & $99.57 \mathrm{M}^{2} \mathrm{H} ;{ }^{3} \mathrm{~K}$ & $0.43 \mathrm{~N}^{2} \mathrm{H} ;{ }^{3} \mathrm{~K}$ & 1.125 & \\
\hline
\end{tabular}

A: $3 d^{2} 4 s^{2}$ configuration; B: $3 d^{3} 4 s$ configuration; C: $3 d^{4}$ configuration; K: $3 d^{3} 5 s$ configuration; L: $3 d^{3} 6 s$ configuration; M: $3 d^{3} 4 d$ configuration; N: $3 d^{3} 5 d$ configuration; ${ }^{*}$ See text. 
Table 2. Fine structure fitted parameters values (in $\cdot \mathrm{cm}^{-1}$ ) for the even-parity levels of V II (Fit) with their uncertainties in parentheses and for comparison their corresponding $a b$ initio values computed by means of the Cowan code (C.C.). See also text.

\begin{tabular}{|c|c|c|c|c|c|c|}
\hline \multirow[t]{2}{*}{ Config. } & \multicolumn{2}{|c|}{$3 d^{2} 4 s^{2}$} & \multicolumn{2}{|c|}{$3 d^{3} 4 s$} & \multicolumn{2}{|c|}{$3 d^{3} 5 s$} \\
\hline & Fit & C.C. & Fit & C.C. & Fit & C.C \\
\hline $\mathrm{E}_{\mathrm{av}}$ & 43,799 (94) & $43,799^{\mathrm{a}}$ & $19,730(30)$ & 18,489 & $84,820(42)$ & 78,881 \\
\hline $\mathrm{F}^{2}(3 \mathrm{~d}, 3 \mathrm{~d})$ & 62,037 (199) & 60,288 & $54,057(66)$ & 54,104 & $57,330(90)$ & 55,519 \\
\hline $\mathrm{F}^{4}(3 \mathrm{~d}, 3 \mathrm{~d})$ & $38,140(271)$ & 37,703 & 31,693 (236) & 33,596 & 34,978 (92) & 34,544 \\
\hline $\mathrm{G}^{2}(3 \mathrm{~d}, \mathrm{~ns})$ & & & 8144 (43) & 8207 & 1339 (35) & 1523 \\
\hline$\zeta_{3 \mathrm{~d}}$ & 200 (17) & 184 & $156(10)$ & 157 & $171(10)$ & 161 \\
\hline$\alpha$ & $50(1)$ & & $50(1)$ & & & \\
\hline$\beta$ & $-130(19)$ & & $-130(19)$ & & & \\
\hline $\mathrm{T}_{\mathrm{s}}$ & & & $4(11)$ & & & \\
\hline $\mathrm{T}_{2}$ & & & $-45(11)$ & & & \\
\hline $\mathrm{T}_{3}$ & & & $-239(13)$ & & & \\
\hline \multirow[t]{2}{*}{ Config. } & \multicolumn{2}{|c|}{$3 d^{4}$} & \multicolumn{2}{|c|}{$3 d^{3} 4 d$} & \multicolumn{2}{|c|}{$3 d^{3} 5 d$} \\
\hline & Fit & C.C. & Fit & C.C. & Fit & C.C. \\
\hline $\mathrm{E}_{\mathrm{av}}$ & 18,687 (13) & 17,691 & 89,766 (32) & 83,105 & $103,174(351)$ & 100,986 \\
\hline$F^{2}(3 d, 3 d)$ & $48,480(56)$ & 47,503 & $56,710(118)$ & 55,559 & $57,837(360)$ & 55,725 \\
\hline$F^{4}(3 d, 3 d)$ & 28,317 (72) & 29,249 & 33,302 (178) & 34,573 & 32,727 (330) & 34,682 \\
\hline$\zeta_{3 \mathrm{~d}}$ & $138(9)$ & 131 & $166(9)$ & 161 & $166(9)$ & 161 \\
\hline$\zeta_{\text {nd }}$ & & & $11(6)$ & 9 & $5(3)$ & 4 \\
\hline$\alpha$ & $50(1)$ & & $7(1)$ & & & \\
\hline$\beta$ & $-130(19)$ & & $-83(16)$ & & & \\
\hline $\mathrm{T}_{2}$ & $-45(11)$ & & & & & \\
\hline $\mathrm{T}_{3}$ & $-239(13)$ & & & & & \\
\hline $\mathrm{F}^{2}(3 \mathrm{~d}, 4 \mathrm{~d})$ & & & 4484 (98) & 5238 & $1910(82)$ & 1847 \\
\hline$F^{4}(3 d, 4 d)$ & & & 2196 (99) & 2230 & 864 (39) & 826 \\
\hline $\mathrm{G}^{0}(3 \mathrm{~d}, 4 \mathrm{~d})$ & & & $1923(26)$ & 2155 & 717 (10) & 1247 \\
\hline $\mathrm{G}^{2}(3 \mathrm{~d}, 4 \mathrm{~d})$ & & & $1551(124)$ & 1927 & $610(50)$ & 922 \\
\hline $\mathrm{G}^{4}(3 \mathrm{~d}, 4 \mathrm{~d})$ & & & $1900(86)$ & 1449 & 758 (34) & 637 \\
\hline
\end{tabular}

${ }^{\mathrm{a}}$ Fixed to the fitted value.

Table 3. Fine structure configuration interaction parameters and for comparison their corresponding ab initio values computed by means of the Cowan code (C.C.).

\begin{tabular}{lccc}
\hline \multicolumn{2}{c}{ Values of main configuration interaction parameters } & \multicolumn{1}{c}{ Fit } & C.C. \\
\hline $3 d^{2} 4 s^{2}-3 d^{3} 4 s$ & $\mathrm{R}^{2}(3 d 3 d, 3 d 4 s)$ & $-1960(192)$ & -4679 \\
$3 d^{2} 4 s^{2}-3 d^{4}$ & $\mathrm{R}^{2}(4 s 4 s, 3 d 3 d)$ & $11869(82)$ & 11839 \\
$3 d^{3} 4 s-3 d^{4}$ & $\mathrm{R}^{2}(3 d 4 s, 3 d 3 d)$ & $-4741(42)$ & -6813 \\
$3 d^{3} 4 d-3 d^{3} 5 d$ & $\mathrm{R}^{2}(3 d 4 d, 3 d 5 d)$ & $2205(173)$ & 2606 \\
& $\mathrm{R}^{4}(3 d 4 d, 3 d 5 d)$ & $1853(253)$ & 1288 \\
$3 d^{3} 4 s-3 d^{3} 4 d$ & $\mathrm{R}^{2}(3 d 4 s, 3 d 4 d)$ & $4564(320)$ & 4109 \\
$3 d^{4}-3 d^{3} 4 d$ & $\mathrm{R}^{2}(3 d 3 d, 3 d 4 d)$ & $6185(380)$ & 8434 \\
& $\mathrm{R}^{4}(3 d 3 d, 3 d 4 d)$ & $3043(583)$ & 5580 \\
$3 d^{3} 5 s-3 d^{3} 4 d$ & $\mathrm{R}^{2}(3 d 5 s, 3 d 4 d)$ & $-320(70)$ & -321 \\
$3 d^{2} 4 s^{2}-3 d^{3} 4 d$ & $\mathrm{R}^{2}(4 s 4 s, 3 d 4 d)$ & $-2303(446)$ & -2946 \\
\hline
\end{tabular}


Table 4. Predicted singlet, triplet and quintet positions for missing experimental energy levels of the configurations mentioned in Table 2.

\begin{tabular}{|c|c|c|c|c|}
\hline Configuration & Designation & $\mathrm{J}$ value & Energy $\left(\mathrm{cm}^{-1}\right)$ & Composition LS (\%) \\
\hline \multirow[t]{2}{*}{$3 d^{4}$} & ${ }^{1} \mathrm{~S}$ & & & \\
\hline & & 0 & 60,212 & 65.7 \\
\hline \multirow[t]{2}{*}{$3 d^{2} 4 s^{2}$} & ${ }^{1} \mathrm{~S}$ & & & \\
\hline & & 0 & 77,196 & 89.6 \\
\hline \multirow[t]{28}{*}{$3 d^{3} 4 d$} & $\left({ }^{4} \mathrm{P}\right){ }^{5} \mathrm{~F}$ & & & \\
\hline & & 1 & 84,407 & 63.7 \\
\hline & & 2 & 84,376 & 65.8 \\
\hline & & 3 & 84,427 & 76.7 \\
\hline & & 4 & 84,503 & 98.0 \\
\hline & & 5 & 84,599 & 98.6 \\
\hline & $\left({ }^{4} \mathrm{P}\right){ }^{5} \mathrm{D}$ & & & \\
\hline & & 0 & 85,178 & 91.8 \\
\hline & & 1 & 85,221 & 89.1 \\
\hline & & 2 & 85,260 & 88.2 \\
\hline & & 3 & 85,291 & 89.3 \\
\hline & & 4 & 85,313 & 91.2 \\
\hline & $\left({ }^{2} \mathrm{G}\right){ }^{3} \mathrm{D}$ & & & \\
\hline & & 1 & 86,241 & 52.5 \\
\hline & & 2 & 86,359 & 30.1 \\
\hline & & 3 & 86,346 & 36.9 \\
\hline & $\left({ }^{4} \mathrm{P}\right){ }^{3} \mathrm{~F}$ & & & \\
\hline & & 2 & 86,536 A.G.E. & 28.8 \\
\hline & & 3 & 86,624 & 36.8 \\
\hline & & 4 & 86,806 & 44.3 \\
\hline & $\left({ }^{2} \mathrm{P}\right){ }^{3} \mathrm{D}$ & & & \\
\hline & & 1 & 87,969 & 67.4 \\
\hline & & 2 & 88,102 & 69.5 \\
\hline & & 3 & 88,420 & 41.8 \\
\hline & $\left({ }^{2} \mathrm{D}\right){ }^{3} \mathrm{G}$ & & & \\
\hline & & 3 & 88,968 & 47.3 \\
\hline & & 4 & 89,005 & 56.2 \\
\hline & & 5 & 89,166 & 57.2 \\
\hline
\end{tabular}




\section{Continued}

$\left({ }^{2} \mathrm{P}\right){ }^{3} \mathrm{~F}$

$\begin{array}{lll}2 & 89,031 & 40.4 \\ 3 & 89,204 & 35.8 \\ 4 & 89,386 & 42.1\end{array}$

$\left({ }^{2} \mathrm{D}\right){ }^{3} \mathrm{~F}$

$\begin{array}{lll}2 & 89,966 & 32.8 \\ 3 & 89,988 & 31.9 \\ 4 & 90,015 & 35.8\end{array}$

$\left({ }^{2} \mathrm{H}\right){ }^{3} \mathrm{~K}$

$\begin{array}{lll}6 & 89,998 & 99.2 \\ 7 & 90,072 & 97.2 \\ 8 & 90,180 & 99.6\end{array}$

$\left({ }^{2} \mathrm{H}\right)^{3} \mathrm{G}$

$\begin{array}{lll}3 & 90,608 & 70.3 \\ 4 & 90,726 & 68.8 \\ 5 & 90,813 & 71.5\end{array}$

$\left({ }^{2} \mathrm{D}\right){ }^{3} \mathrm{P}$

$\begin{array}{lll}0 & 90,707 & 59.6 \\ 1 & 90,840 & 56.7 \\ 2 & 91,075 & 48.3\end{array}$

$\left({ }^{2} \mathrm{D}\right){ }^{3} \mathrm{D}$

$\begin{array}{lll}1 & 91,494 & 42.8 \\ 2 & 91,440 & 35.9 \\ 3 & 91,354 & 44.5\end{array}$

$\left({ }^{2} \mathrm{H}\right){ }^{3} \mathrm{H}$

$\begin{array}{lll}4 & 91,203 & 85.1 \\ 5 & 91,278 & 86.1 \\ 6 & 91,351 & 87.2\end{array}$

$\left({ }^{2} \mathrm{D}\right){ }^{1} \mathrm{~S}$

$\begin{array}{lll}0 & 94,473 & 71.6\end{array}$

$\left({ }^{2} \mathrm{P}\right){ }^{1} \mathrm{P}$

$\begin{array}{lll}1 & 87,655 & 51.5\end{array}$

$\left({ }^{2} \mathrm{D}\right){ }^{1} \mathrm{P}$

$\begin{array}{lll}1 & 89,006 & 49.4\end{array}$

$\left({ }^{2} \mathrm{D}\right){ }^{1} \mathrm{D}$

2

90,040

67.8

$\left({ }^{2} \mathrm{P}\right){ }^{1} \mathrm{D}$ 


\section{Continued}

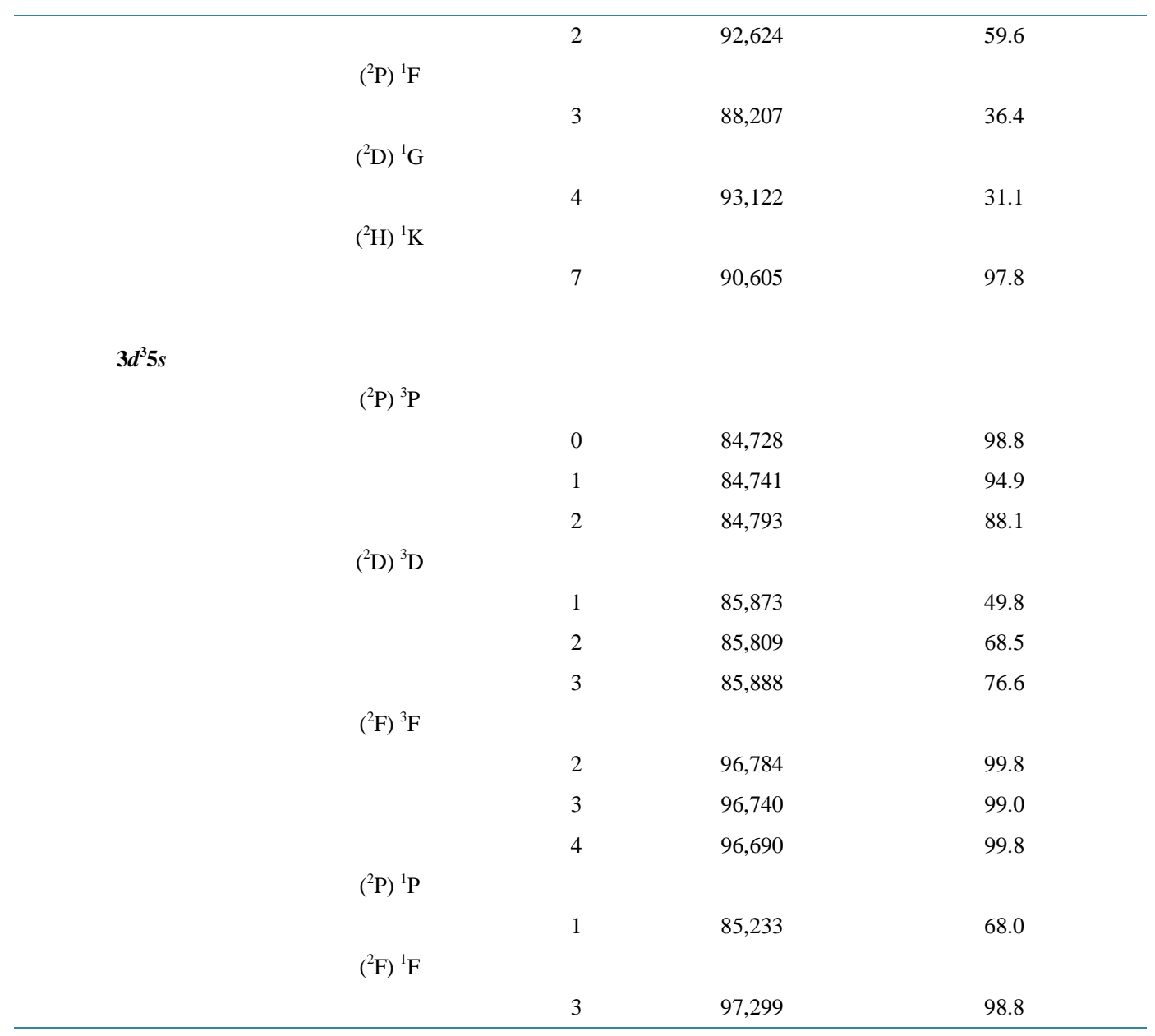

A.G.E.: already given experimentally.

A good fit, with a root mean square uncertainty of $6.2 \mathrm{MHz}$ was obtained. The values of the model space hfs parameters, quoted with their uncertainties, are presented in Table 5 . In order to check the validity of these fitted parameters we have compared some of them to those computed using values obtained by means of the Cowan code. For example one can use the well-established relation $a_{n l}^{\kappa k}(\mathrm{MHz})=2 \mu_{0} \mu_{B} \mu_{I}\left\langle r^{-3}\right\rangle_{n l}^{\kappa k} / 4 \pi I=95.4128 g_{I}\left\langle r^{-3}\right\rangle_{n l}^{\kappa k}$. Using the values of line 2 of Table 6, knowing that the nuclear spin and magnetic dipole moment of ${ }^{51} \mathrm{~V}$ are equal respectively to $7 / 2$ and $5.1485 \mu_{\mathrm{n}}$ one gets the $a_{3 d}^{01}$ values of line 3 of Table 6 which are on the whole close to the experimental ones, located in line 1 of the same Table. This confirms the well-founded basis of our work.

To check the value of the most influential hfs-deduced parameter, $a_{4 s}^{10}\left(3 d^{3} 4 s\right)$, it is interesting to compare the ratio $\frac{a_{4 s}^{10}\left(3 d^{4} 4 s\right)}{a_{4 s}^{10}\left(3 d^{3} 4 s\right)}=\frac{2535}{4519.76}=0.56$ relative to V I [13] and V II (this work) with $\frac{a_{4 s}^{10}\left(3 d^{3} 4 s\right)}{a_{4 s}^{10}\left(3 d^{2} 4 s\right)}=\frac{-487}{-836.27}=0.58$ relative to Ti I [14] and Ti II [7]. Since these ratios are very close for these two neighbour elements in the Periodic Table, we can conclude that the deduced $a_{4 s}^{10}\left(3 d^{3} 4 s\right)$ value for V II is really satisfactory. To extract magnetic dipole A-values from experimental hfs splitting the electric quadrupole hfs B factors preferably were fixed deliberately to zero in [12] because the electric quadrupole moment of ${ }^{51} \mathrm{~V}$ is small: $-0.05 \mathrm{~b}$. In this case it is not useful to compute $b_{n l}^{\kappa k}$ values since it is not possible to make comparisons between experimental and theoretical $b_{n l}^{\kappa k}$ values.

In Table 7 we have listed the measured hfs constants A given in $\mathrm{MHz}$, used in our fitting procedure. In this 
Table 5. The fitted hfs many-body parameter values in $\mathrm{MHz}$ for the model space $(3 d+4 s)^{4}$. The uncertainties given in parentheses are the standard deviations.

\begin{tabular}{cccc}
\hline$a_{3 d}^{01}$ & $498.71(6.62)$ & $\mathrm{a}_{1}$ & $177.04(6.45)$ \\
$a_{3 d}^{12}$ & $469.24(23.02)$ & $\mathrm{a}_{2}$ & $51.34(1.87)$ \\
$a_{3 d}^{10}$ & $-14.86(9.33)$ & $\mathrm{a}_{3}$ & $34.17(1.24)$ \\
$a_{4 s}^{10}$ & $4519.76(130.78)$ & $\mathrm{a}_{9}$ & $813.26(36.93)$ \\
$a_{1 c}^{12}$ & $44.50(19.81)$ & $\mathrm{a}_{11}$ & $-3617.15(135.90)$ \\
\hline
\end{tabular}

$\mathrm{a}_{4}=\mathrm{a}_{5}=\mathrm{a}_{6}=\mathrm{a}_{7}=\mathrm{a}_{8}=\mathrm{a}_{10}=0.00$ (fixed).

Table 6. Comparison between fitted and calculated hfs many-body parameter $a_{3 d}^{01}$ in MHz. Radial integrals are computed by means of the pseudo-relativistic Cowan code.

\begin{tabular}{|c|c|c|c|}
\hline Parameter & $3 d^{2} 4 s^{2}$ & $3 d^{3} 4 s$ & $3 d^{4}$ \\
\hline$a_{3 d}^{01} \quad$ (Fit) (MHz) & 427.90 & 357.09 & 286.27 \\
\hline$\left\langle\mathrm{r}^{-3}\right\rangle_{3 d}$ (a.u.) & 3.018 & 2.615 & 2.233 \\
\hline$a_{3 d}^{01} \quad(\mathrm{Cal})(\mathrm{MHz})$ & 423.58 & 367.02 & 313.41 \\
\hline
\end{tabular}

Table 7. Our computed hfs A-constants of V II (in MHz), compared with those obtained experimentally by Armstrong et al. [10].

\begin{tabular}{|c|c|c|c|c|}
\hline Energy $\left(\mathrm{cm}^{-1}\right)$ & \multicolumn{2}{|c|}{ Designation [1] } & $\mathrm{A}_{\exp }(\mathrm{MHz})[10]$ & $\mathrm{A}_{\text {cal }}(\mathrm{MHz})$ This work \\
\hline 18269.49 & $3 d^{4}$ & $a^{3} D$ & -38.51 & -36.48 \\
\hline 32299.27 & $3 d^{4}$ & $\mathrm{~d}^{3} \mathrm{P}$ & -73.33 & -69.03 \\
\hline \multicolumn{5}{|l|}{$\mathbf{J}=\mathbf{2}$} \\
\hline 13490.89 & $3 d^{4}$ & $\mathrm{~b}^{3} \mathrm{~F}$ & 481.96 & 480.84 \\
\hline 13594.73 & $3 d^{3} 4 s$ & $a^{5} \mathrm{P}$ & 1096.69 & 1098.88 \\
\hline 18293.88 & $3 d^{4}$ & $a^{3} D$ & 488.08 & 453.85 \\
\hline 30267.47 & $3 d^{4}$ & $d^{3} F^{*}$ & 397.31 & 424.89 \\
\hline 30673.08 & $3 d^{3} 4 s$ & $c{ }^{3} F^{*}$ & -250.92 & -269.78 \\
\hline 32040.64 & $3 d^{4}$ & $\mathrm{~d}^{3} \mathrm{P}$ & 0 (fixed) & -2.72 \\
\hline 44657.94 & $3 d^{2} 4 s^{2}$ & $\mathrm{C}^{1} \mathrm{D}$ & 365.79 & 379.22 \\
\hline \multicolumn{5}{|l|}{$\mathbf{J}=\mathbf{3}$} \\
\hline 13542.67 & $3 d^{4}$ & $\mathrm{~b}^{3} \mathrm{~F}$ & 250.91 & 255.18 \\
\hline 13741.61 & $3 d^{3} 4 s$ & $a^{5} \mathrm{P}$ & 840.96 & 850.03 \\
\hline 16340.97 & $3 d^{3} 4 s$ & $b^{3} G$ & 138.66 & 148.39 \\
\hline 18353.88 & $3 d^{4}$ & $a^{3} \mathrm{D}$ & 502.93 & 494.22 \\
\hline 26839.77 & $3 d^{4}$ & $\mathrm{a}^{1} \mathrm{~F}$ & 301.10 & 293.02 \\
\hline 30306.38 & $3 d^{4}$ & $d^{3} F^{*}$ & 332.71 & 331.54 \\
\hline 30641.76 & $3 d^{3} 4 s$ & $c^{3} F^{*}$ & 414.24 & 422.43 \\
\hline 34228.82 & $3 d^{3} 4 s$ & $\mathrm{~b}^{1} \mathrm{~F}$ & 355.08 & 352.80 \\
\hline
\end{tabular}




\begin{tabular}{ccccc} 
Continued & \multicolumn{5}{c}{} \\
\hline $\mathbf{J}=\mathbf{4}$ & & & & \\
13608.96 & $3 \mathrm{~d}^{4}$ & $\mathrm{~b}{ }^{3} \mathrm{~F}$ & 171.4 & 174.57 \\
16421.51 & $3 \mathrm{~d}^{3} 4 \mathrm{~s}$ & $\mathrm{~b}^{3} \mathrm{G}$ & 423.32 & 416.55 \\
30318.55 & $3 \mathrm{~d}^{4}$ & $\mathrm{~d}^{3} \mathrm{~F}^{*}$ & 434.52 & 394.42 \\
30613.92 & $3 \mathrm{~d}^{3} 4 \mathrm{~s}$ & $\mathrm{c}^{3} \mathrm{~F}^{*}$ & 591.70 & 623.83 \\
38517.06 & $3 \mathrm{~d}^{2} 4 \mathrm{~s}^{2}$ & $\mathrm{e}^{3} \mathrm{~F}$ & 276.67 & 263.47 \\
$\mathbf{J}=\mathbf{5}$ & & & 436.10 & 452.52 \\
14655.63 & $3 \mathrm{~d}^{4}$ & $\mathrm{a}^{3} \mathrm{G}$ & 498.41 & 495.91 \\
16533.00 & $3 \mathrm{~d}^{3} 4 \mathrm{~s}$ & $\mathrm{~b}{ }^{3} \mathrm{G}$ & & \\
\hline
\end{tabular}

*: See text.

Table we inserted also our computed values which confirm totally the Armstrong, Rosner and Holt experimental data [12] which are sometimes different from those of Arvidsson [15]. As regards the $3 d^{2} 4 s^{2}$ e ${ }^{3} F_{4}(38517.06$ $\mathrm{cm}^{-1}$ ) hfs value we reject $\mathrm{A}=-351.28$ and we keep $\mathrm{A}=276.67 \mathrm{MHz}$ since two values were proposed in [12] (owing to an unavoidable ambiguity in $\Delta \mathrm{J}=0$ transitions).

\section{Conclusion}

Parametric fs studies including configuration interactions have been carried out for five interacting configurations of V II. We furthermore propose predicted energy level values for missing experimental ones up to $100,000 \mathrm{~cm}^{-1}$ for further investigations. One can also note that calculated Landé $\mathrm{g}_{\mathrm{J}}$-factors were also in good agreement with the experimental ones. Unfortunately the latter are not as numerous as we might have expected and thus our work to check level assignments became more difficult. We give for the first time the hfs manybody parameter values with good accuracy for the model space $(3 d+4 s)^{4}$, taking advantage of the accurate work done in [10]. This provides better predictions for still unknown levels. The conclusive comparison between the experimental and calculated hfs A-constants, given in Table 7, provides a good check on the quality of the wave functions obtained by the least squares fit of the fine structure, used to determine the expansions of hfs A-constants in intermediate coupling. Very recently the spectrum of V II has been recorded by FTS and thirtynine of the additional eighty-five high levels published by Iglesias et al. [3] have been confirmed or revised, and four of their missing levels have been found [16] as regards even-parity levels. One can note in our Table 1 a total agreement with the assignments of these four new levels given in this interesting work.

\section{References}

[1] Meggers, W.F. and Moore, C.E. (1940) Journal of Research of the National Bureau of Standards (US), $25,83$.

[2] Sugar, J. and Corliss, C.H. (1985) Journal of Physical and Chemical Reference Data, 14, 1-664. http://dx.doi.org/10.1063/1.555747

[3] Iglesias, L., Cabeza, M.I., Garcia-Riquelme, O. and Rico, F.R. (1987) Optica Pura y Aplicada, 2, 137.

[4] Bouazza, S. (2011) International Journal of Quantum Chemistry, 111, 3000-3007. http://dx.doi.org/10.1002/qua.22614

[5] Bouazza, S. (2012) International Journal of Quantum Chemistry, 112, 470-477. http://dx.doi.org/10.1002/qua.22974

[6] Bouazza, S. (2012) Physica Scripta, 86, 015302. http://dx.doi.org/10.1088/0031-8949/86/01/015302

[7] Bouazza, S. (2013) Physica Scripta, 87, 045301. http://dx.doi.org/10.1088/0031-8949/87/04/045301

[8] Bouazza, S. (2013) Physica Scripta, 87, 035302. http://dx.doi.org/10.1088/0031-8949/87/03/035302

[9] Cowan, R.D. (1981) The Theory of Atomic Structure and Spectra. University of California Press, Berkeley.

[10] Armstrong, N.M.R., Rosner, S.D. and Holt, R.A. (2011) Physica Scripta, 84, 055301. http://dx.doi.org/10.1088/0031-8949/84/05/055301

[11] Dembczynski, J. (1996) Physica Scripta, 65, 88.

[12] Bouazza, S., Dembczynski, J., Stachowska, E., Szawiola, G. and Ruczkowski, J. (1998) European Physical Journal D, 
4, 39.

[13] Palmeri, P., Biémont, E., Quinet, P., Dembczynski, J., Szawiola, G. and Kurucz, R.L. (1997) Physica Scripta, 55, 586. http://dx.doi.org/10.1088/0031-8949/55/5/011

[14] Aydin, R., Stachowska, E., Johann, U., Dembzynski, J., Unkel, P. and Ertmer, W. (1990) Zeitschrift für Physik D, 15, 281.

[15] Arvidsson, K. (2003) Master’s Thesis Lund Observatory. Lund University, Sweden.

[16] Thorne, A.P., Pickering, J.C. and Semeniuk, J.I. (2013) The Astrophysical Journal, 207, 13. http://dx.doi.org/10.1088/0067-0049/207/1/13 